\title{
PHOTOTHERMAL ABLATION OF POLYSTYRENE FILM BY 248 NM EXCIMER LASER IRRADIATION: A MECHANISTIC STUDY BY TIME-RESOLVED MEASUREMENTS
}

\author{
YASUYUKI TSUBOI, SHIN-ICHI SAKASHITA, KOJI HATANAKA, \\ HIROSHI FUKUMURA and HIROSHI MASUHARA* \\ Department of Applied Physics, Osaka University, \\ Suita, Osaka 565, Japan
}

(Received 25 July, 1995)

\begin{abstract}
Laser ablation mechanism at $248 \mathrm{~nm}$ irradiation of polystyrene film was investigated and discussed. An ablation threshold was determined by etch depth measurement and nanosecond photographic observation. Temperature at the threshold was evaluated to be $370^{\circ} \mathrm{C}$ by using an effective absorption coefficient which was confirmed by transmission measurement of the excitation pulse. The temperature was in good agreement with that of thermal degradation of the polymer, showing that $248 \mathrm{~nm}$ ablation of polystyrene was brought about by a photothermal process. Nanosecond photography upon the ablation could be well interpreted from the photothermal viewpoint. Moreover, dynamics of excited species revealed by nanosecond time-resolved luminescence measurements was inferred in relation to the ablation.
\end{abstract}

KEY WORDS: Excimer laser ablation, Polystyrene, Photothermal mechanism, Time-resolved spectroscopy, Time-resolved photography.

\section{INTRODUCTION}

Since the first report of polymer ablation by UV laser irradiation appeared in $1982,{ }^{1,2}$ considerable efforts have been given to reveal fundamental mechanisms governing the phenomenon in terms of nonlinear photo-processes. According to the preceding numerous investigations, dominant processes responsible for ablation are now considered in terms of photochemical ${ }^{3,4}$ and photothermal ${ }^{5,6}$ mechanisms. Both mechanisms are of course interrelated with each other and cannot be separated in real processes, hence their relative contribution to the ablation have been a subject of controversy. Indeed, mechanistic aspects of laser ablation have been widely investigated for various organic polymers in the past decade. Most researchers have dealt with poly(methyl methacrylate) (PMMA), ${ }^{7,8}$ polyimide, ${ }^{9,10}$ and poly(ethylene terephthalate) ${ }^{11}$ as ablation targets mainly due to scientific interest as laser-matter interaction and high potential for industrial applications. On the contrary, there have been few systematic studies focusing onto ablation of polystyrene,

\footnotetext{
*To whom all correspondence should be addressed.
} 
especially at $248 \mathrm{~nm}$ excitation, although the polymer exhibits excellent electric as well as thermal properties as an organic material. The polystyrene has phenyl groups as a chromophore whose photophysical/photochemical primary processes are well understood, ${ }^{12}$ hence the polymer is quite suitable for obtaining further insights into ablation mechanisms.

One mechanistic approach from photochemical viewpoint is to correlate ablation behavior with photochemical reactivity of light-absorbing chromophore in the polymer. Recently we reported that liquid benzene derivatives such as toluene, benzyl chloride, chlorobenzene, etc., showed very interesting ablation behavior by $248 \mathrm{~nm}$ laser irradiation. ${ }^{13}$ In this system, namely, we confirmed a direct relation between the ablation threshold and the molecular photochemical reactivity: As the reactivity increased, the threshold value decreased. Note that the polystyrene has the same chromophore as the benzene derivatives (phenyl group), hence it is expected that the similar mechanistic understanding is possible. On the other hand, however, $248 \mathrm{~nm}$ ablation has been frequently concluded as photothermal mechanism; for example, neat PMMA and doped one with aromatic molecules (biphenyl, pyrene, etc.). ${ }^{14,15}$ Thermal energy is dissipated through "Cylic multiphotonic absorption" of laser light. ${ }^{16}$ Namely, the excited singlet and triplet states are formed and absorb the light competetively with the ground state. As the formed higher excited states have a short lifetime and relax to the original excited state, the recovered latter states absorb the light again, and so on. Due to the repetitive absorption during the laser pulse, light absorbing molecules are considered to be a molecular heater. Whether such phothermal mechanism is involved in polystyrene ablation or not is one of the present purposes.

All these results contrasting each other strongly imply that fundamental mechanisms responsible for ablation of organic materials are quite complicated to understand, especially at $248 \mathrm{~nm}$ excitation. Hence, it seems intriguing to compare the polystyrene ablation with that of liquid benzene derivatives as well as doped polymer ablation. Along the line, we have investigated a mechanism of $248 \mathrm{~nm}$ ablation of polystyrene by means of etch depth, transmittance of excitation pulse, timeresolved photographic, and nanosecond luminescence measurements. The results obtained by these procedures are summarized, compared with the previous reports, and shown to be well interpreted in terms of photothermal ablation.

\section{EXPERIMENTAL}

Polystyrene (Wako, degree of polymerization $=1600 \sim 1800)$ was reprecipitated from benzene-methanol, and dried under low temperature. Chlorobenzene solution of $12-15 \mathrm{wt} \%$ polystyrene was coated onto quartz substrate by a spinner (Mikasa, 1H-D3) to produce films with $3-4 \mu \mathrm{m}$ thickness. Thick films $(20 \mu \mathrm{m})$ were also prepared by casting the solution, which were used only for the spectroscopic measurements. These films were dried in a vacuum oven over a night before measurements.

A KrF excimer laser (Lambda Physik, LEXTRA 200) provided a $248 \mathrm{~nm}$ pulse (FWHM $\sim 20 \mathrm{~ns}$ ) which was used as an excitation light. The laser fluence was 
adjusted by using attenuators, and measured by a power meter (Gentec, ED-500). Etch depth of the irradiated films was measured by a depth profiler (Nihon Shinku, DEKTAK 3).

The details of experimental set-up for transmittance measurement of an excitation pulse were described previously, ${ }^{17}$ which is briefly introduced here. A fraction of incident laser light and transmitted one through a sample were detected by two photodiodes (Hamamatsu, S 1722-02), respectively. The outputs were transferred through a digital oscilloscope (Hewlet Packard, HP54510A, $250 \mathrm{~Hz}$ ) to a computer, by which absorbance change at $248 \mathrm{~nm}$ was calculated.

The experimental set-up for nanosecond imaging of plumes ejected by ablation was similar to that described elsewhere ${ }^{18}$ except for the sample unit. The essential of this technique is employing visible fluorescence of dye solution with nanosecond duration as back illumination. In the present case, dichloromethane solution of Rhodamine 101 was excited to supply the illumination by the second harmonic of a $\mathrm{Nd}^{3+}$ : YAG laser (JK laser, HY750) coupled electrically with the excimer laser. The temporal resolution of the system, regulated by the duration time of the Rhodamine fluorescence, was about $20 \mathrm{~ns}$.

For the luminescence spectroscopy, the streak camera (Hamamatsu, C3140) system was adopted as done in our previous studies, ${ }^{19}$ by which we could measure spectra of some transient species within/somewhat after the excitation pulse.

For all the time-resolved measurements, the temporal origin was defined as the time when an excitation pulse reached its maximum. Also, fresh surface was always irradiated and measured to avoid contributions of photo-induced morphological changes and the photochemical products.

\section{RESULTS AND DISCUSSION}

As the first step etching of polystyrene film at $248 \mathrm{~nm}$ excitation was examined. Figure 1 shows a laser fluence dependence of etch depth. One can easily recognize that significant etching is achieved beyond a laser fluence of $130 \mathrm{~mJ} / \mathrm{cm}^{2}$, which was adopted as the ablation threshold. This value is not inconsistent with the reported value, which is discussed later. When the fluence exceeds $\sim 300 \mathrm{~mJ} / \mathrm{cm}^{2}$, however, a saturation was observed. The saturation tendency of etch depth has been frequently reported for other polymer ablation, and one possible explanation is the shielding effect of laser light by ablation plume ${ }^{20}$ as well as bleaching of the ligh-absorbing molecule. $^{21}$

To confirm the ablation threshold and to examine a possible contribution of the shielding effect, nanosecond photographs upon laser ablation were taken at various fluences. The morphological changes, signs of ablation, were not detected at a fluence below $\sim 110 \mathrm{~mJ} / \mathrm{cm}^{2}$ and observed at $170 \mathrm{~mJ} / \mathrm{cm}^{2}$, which confirms the value of ablation threshold of $130 \mathrm{~mJ} / \mathrm{cm}^{2}$ determined by the etch depth measurement. One characteristic in the imaging results around the threshold was that apparent ablation plume was observed only slightly. The results are in distinctly contrast with that of PMMA ${ }^{22,23}$ and doped $\mathrm{PMMA}^{23}$ where the ablation plume is apparently observed. In PMMA systems, ablation products are mainly composed of MMA 


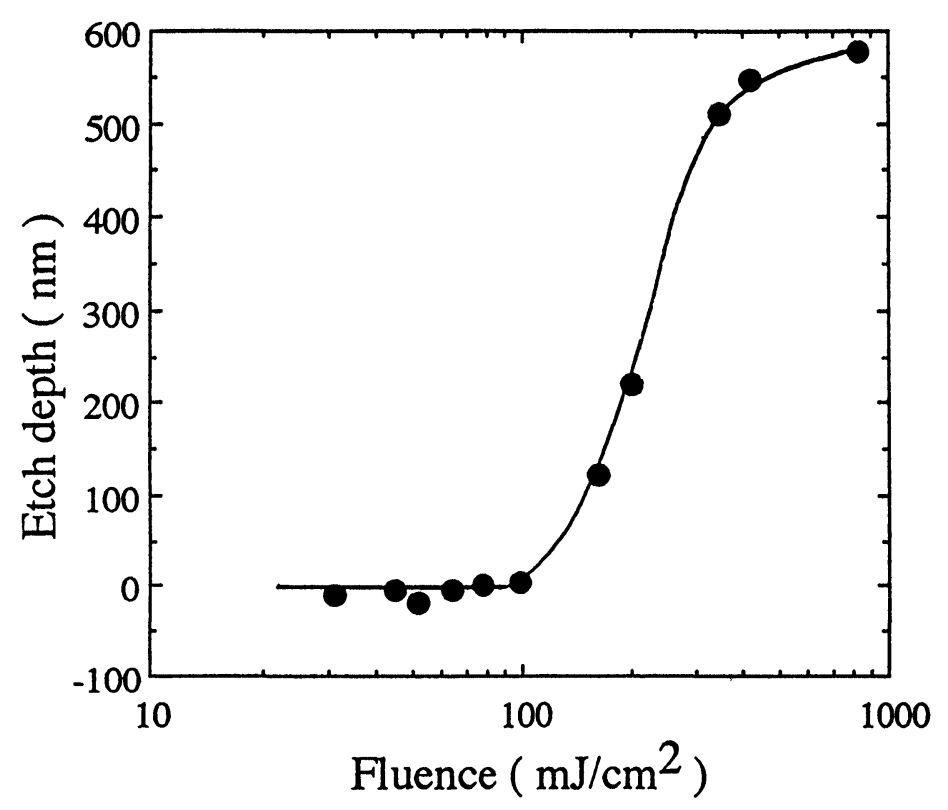

Figure 1 Ablation etch depth as a function of laser fluence.

oligomers (or polymer debris), ${ }^{24}$ hence the large fragments of polymers can be detected visibly by imaging apparatuses with the spatial resolution of $\sim 10 \mu \mathrm{m}$. The present imaging results on polystyrene ablation strongly suggest that the formation of low-molecular weight oligomers and monomer styrene induces ablation. This speculation would be valid, since the plume in $248 \mathrm{~nm}$ polystyrene ablation is composed, in a large part, of monomer styrene which was confirmed already by time-of-flight mass spectrometric technique. ${ }^{25}$ The plume ejection at $170 \mathrm{~mJ} / \mathrm{cm}^{2}$ were observed quite slightly in the flames at $100 \mathrm{~ns}$ and $1 \mu \mathrm{s}$.

Time-resolved transmittance measurement of excitation pulse was carried out to determine an effective absorption coefficient, $\alpha_{\text {eff }}$, at the laser wavelength, which is a key parameter to understand ablation mechanisms. The absorption coefficient could often change within the excitation pulse width due to bleaching of the ground state and densely generated excited species by intense laser irradiation leading to ablation. ${ }^{15,16}$ Transient absorbance at the $248 \mathrm{~nm}$ excitation wavelength is given in Figure 2. At the fluences of 100 (not shown in the figure.) and $200 \mathrm{~mJ} / \mathrm{cm}^{2}$, the absorbance held almost constant, values of which were identical with that obtained by a steady-state measurement. Therefore, we can adopt a value of $3450 \mathrm{~cm}^{-1}$, which was determined by the steady-state measurements, as $\alpha_{\text {eff }}$ even for the case of an intense excitation around the ablation threshold. On the other hand, in Figure 2 for $300 \mathrm{~mJ} / \mathrm{cm}^{2}$, a rise of transient absorbance was observed in the late part of the laser pulse, which would be ascribed to the plume shielding by excitation pulse. Lemoine also reported the shielding at $300 \mathrm{~mJ} / \mathrm{cm}^{2}$ by investigating the reflected pulse, ${ }^{26}$ which is consistent with our result. We consider the saturation in etch depth is arising from the shielding effect. 


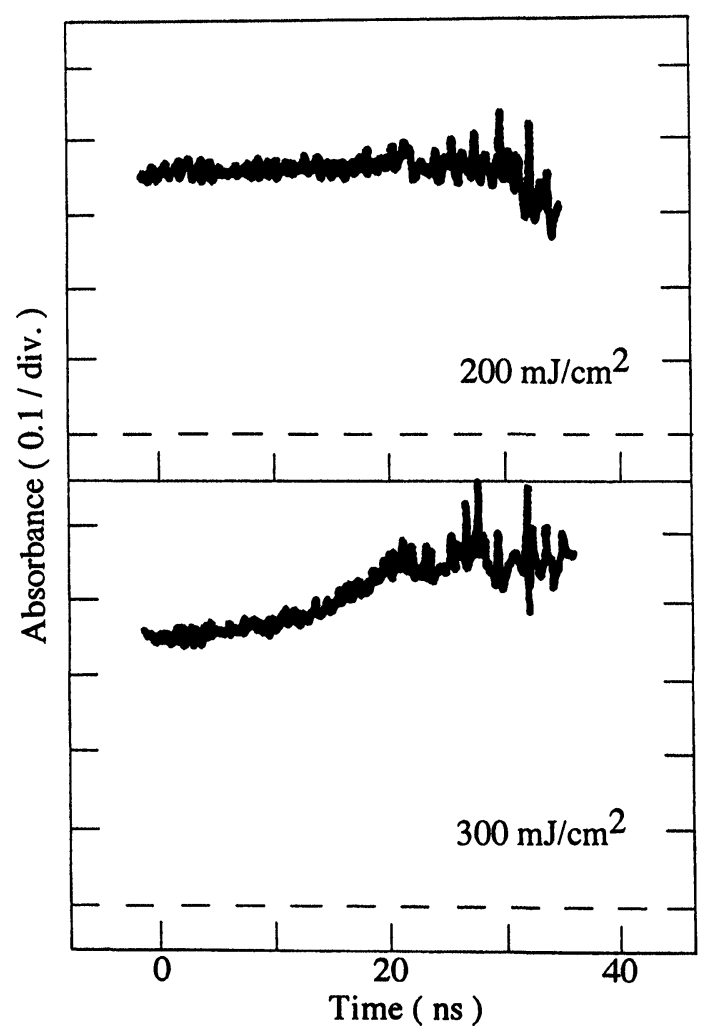

Figure 2 Transient absorbance at the excitation wavelength during the laser. The laser fluence is shown in the figure.

As the effective absorption coefficient at the threshold was confirmed experimentally, we can estimate surface temperature by using the following well-known equation on the assumption that the absorbed energy is entirely converted into heat. ${ }^{27,28}$

$$
\mathrm{T}_{\mathrm{th}}=\mathrm{T}_{\mathrm{r}}+\mathrm{F}_{\mathrm{th}} \alpha_{\mathrm{eff}} / \gamma \mathrm{C}
$$

where $T_{\text {th }}$ is temperature at the threshold, $T_{r}$ is room temperature of $20^{\circ} \mathrm{C}, \mathrm{F}_{\text {th }}$ is the ablation threshold, and $\gamma$ and $C$ are the density $\left(1.06 \mathrm{~g} / \mathrm{cm}^{3}\right)$ and specific heat $(1.21$ $\mathrm{J} / \mathrm{Kg}$ ) of the polymer, respectively. ${ }^{29}$ By using the experimentally confirmed data of $F_{t h}=130 \mathrm{~mJ} / \mathrm{cm}^{2}$ and $\alpha_{\text {eff }}=3450 \mathrm{~cm}^{-1}$, the $T_{\text {th }}$ was evaluated to be $370{ }^{\circ} \mathrm{C}$. This temperature is well consistent with that of thermal degradation of the polystyrene $\left(\sim 350{ }^{\circ} \mathrm{C}^{30}, \sim 300{ }^{\circ} \mathrm{C}^{31}\right)$, suggesting that the ablation is brought about photothermally. The monomer styrene is reported to be the main product in pyrolysis of polystyrene, ${ }^{30,31}$ supporting the photothermal ablation. Furthermore, we could interpret difficult observation of ejected plumes in nanosecond photographs. In using eq. (1), we have neglected temperature dependencies of $\gamma$ and $C$. With rising temperature, $\gamma$ decreases while $\mathrm{C}$ increases accordingly, hence we consider the two factors cancel each other. 
Nanosecond time-resolved luminescence spectra were measured to obtain information on dynamics of transient species upon the ablation. Figure 3 shows the spectroscopic results at various laser fluences. At 28,130 , and $420 \mathrm{~mJ} / \mathrm{cm}^{2}$, we mainly observed broad excimer fluorescence whose maximum was located around $330 \mathrm{~nm}$, with a weak overlap of monomer fluorescence of phenyl group around 280 $\mathrm{nm} .{ }^{31}$ It is noteworthy here that monomer styrene has a fluorescence band maximum at $320 \mathrm{~nm}$, which might superimpose the excimer band especially at 420 $\mathrm{mJ} / \mathrm{cm}^{2}$. In solid polystyrene, the phenyl monomer fluorescence is quenched rapidly with a time constant of $\sim 10 \mathrm{ps}$ due to fast energy migration to stable trap sites or slight rotational motion of phenyl groups to generate the excimer. ${ }^{33}$ Note that little monomer fluorescence was observed by a steady state measurement. Our detection of monomer fluorescence should be ascribed to a peculiar behavior due to dense excitation. One possible explanation is due to $\mathrm{S}_{1}-\mathrm{S}_{1}$ / excimer-excimer annihilation processes where the relative contribution of excimer fluorescence to monomer one is reduced as the intensity increases. ${ }^{34}$ Furthermore, the processes emit heat energy, thus the ratio of monomer fluorescence intensity to that of excimer increases with

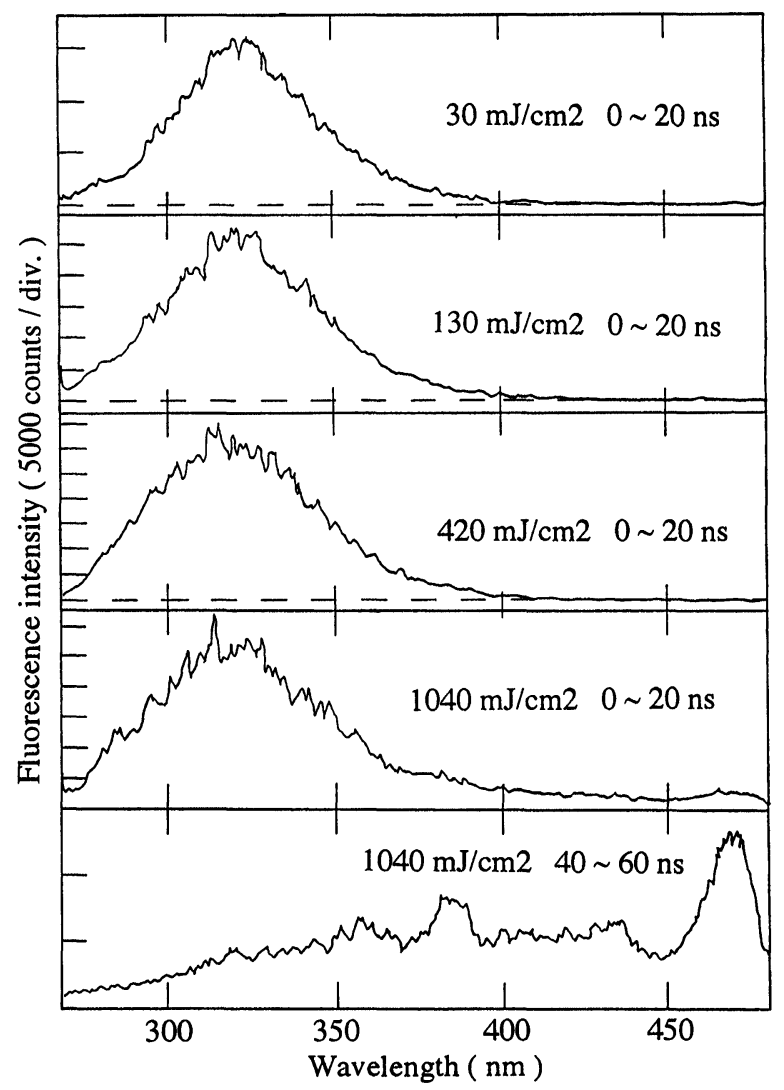

Figure 3 Nanosecond time-resolved luminescence spectra at various fluences. Gate time is given in the figure. 
the fluence as shown in Figure 4. Under the condition of intense light excitation, additional processes such as multi-photon ionization followed by electron capture by the phenyl group would be involved, which may also contribute to heat generation..$^{35}$

In Figure 3 are also shown time-resolved spectra at the fluence $\left(1000 \mathrm{~mJ} / \mathrm{cm}^{2}\right)$ highly above the threshold. In the figure, we distinctly observed several radical fluorescence at the late stage of $40 \sim 60 \mathrm{~ns}$. The sharp bands at $380 \mathrm{~nm}$ and $470 \mathrm{~nm}$ were assigned to $\mathrm{CN}$ and $\mathrm{C}_{2}$ radicals (so-called Swan band), respectively. ${ }^{35}$ These radicals were detected at fluences $>500 \mathrm{~mJ} / \mathrm{cm}^{2}$. The temperature at $1000 \mathrm{~mJ} / \mathrm{cm}^{2}$ was estimated to be $\sim 2000{ }^{\circ} \mathrm{C}$ by using eq. (1) which may be an upper limit because the plume shielding effect was not taken into account. At such high temperature, the polymer undergoes rapid pyrolysis giving radicals of small molecules or atoms. ${ }^{30,31}$ The $\mathrm{CN}$ radical was generated through a reaction between the carbon radical and $\mathrm{N}_{2}$ molecule in atmosphere. Thus, the spectroscopic results can be well interpreted as dynamic behavior of thermal decomposition. The excimer fluorescence intensity obtained by integration through the time range of $-30 \sim 200 \mathrm{~ns}$ was plotted against the fluence in Figure 5, showing considerable saturation tendency even below the threshold. The spectroscopic results that no radical fluorescence observation below/above the threshold and the apparent saturation of the fluorescence intensity do not contradict with the postulation that absorbed light energy is entirely converted into heat in eq. (1). The pyrolysis leading to radical formation should take place at late stages, therefore, we observed no radical fluorescence around the threshold.

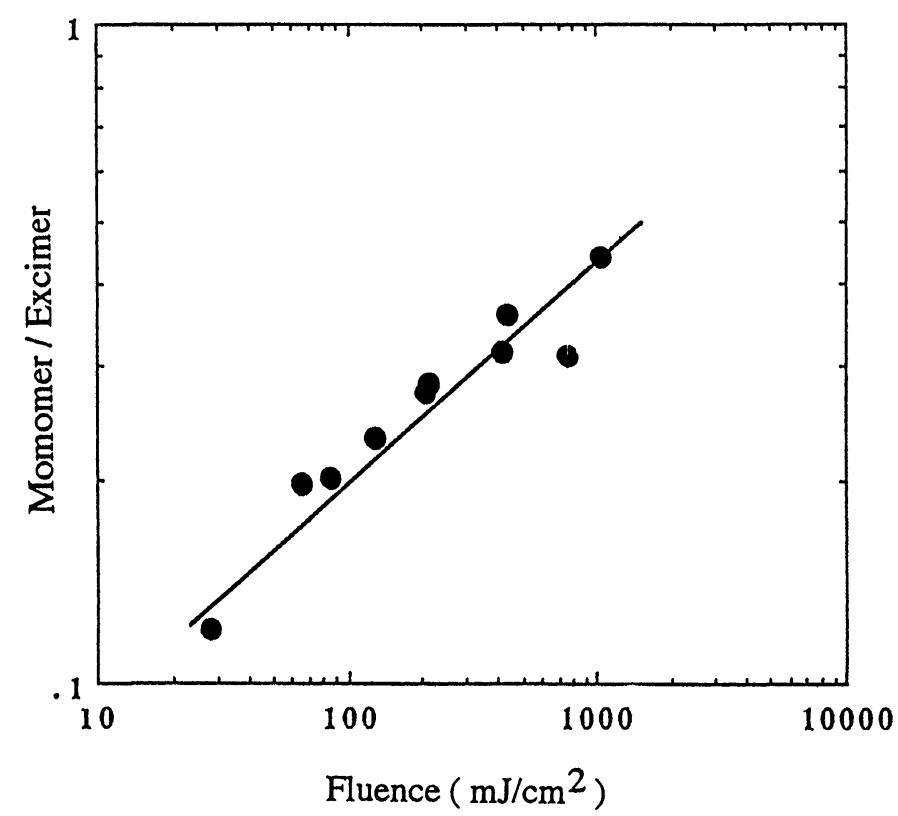

Figure 4 Ratio of fluorescence intensity of monomer to that of excimer as a function of laser fluence. The data in the figure are taken from total luminescence spectra $(-30 \sim 200 \mathrm{~ns})$. 


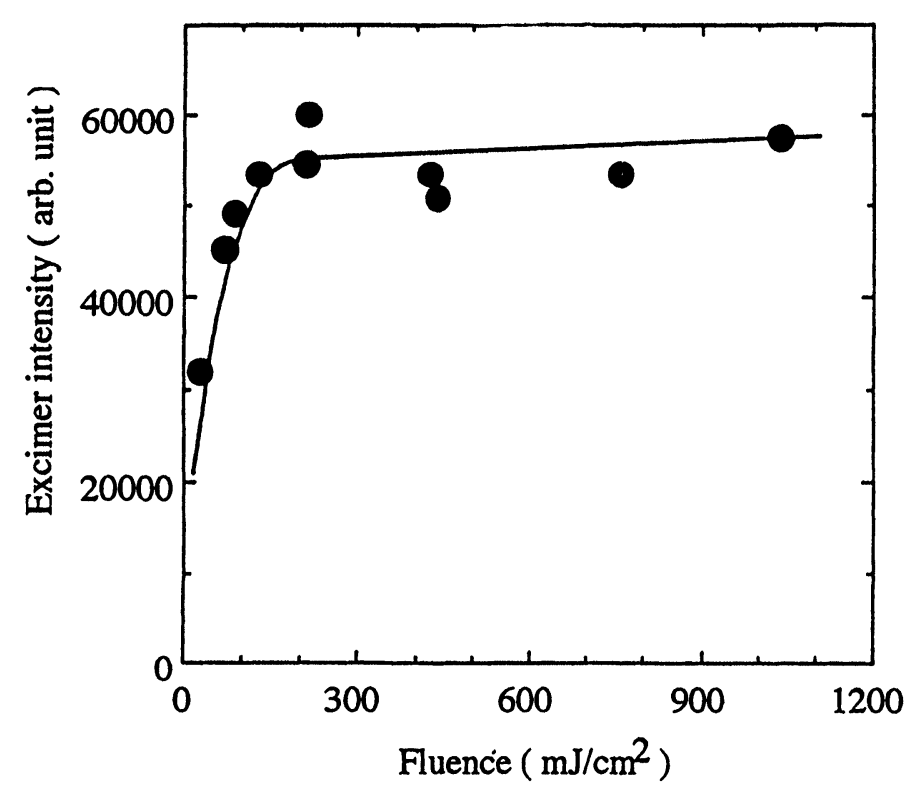

Figure 5 Excimer fluorescence intensity as a function of laser fluence.

Here we discuss a mechanism whereby the heat energy is efficiently generated. According to our previous works on the ablation of polymers doped with aromatic molecules, the dominant process responsible for the conversion of light energy into heat is considered to be the "cyclic multiphotonic absorption mechanism", ${ }_{15,16,37}$ outline of which is introduced in the above. By the intense irradiation, most of dopants are excited to their transient states (namely, lowest excited triplet states, $T_{1}$, etc.). then the absorption by the excited states $\left(T_{n}<--T_{1}\right.$, etc.) is appreciable. It is generally accepted that relaxation processes between electronically excited states $\left(S_{n}-S_{1}, T_{n}-T_{1}\right.$, etc.) are quite rapid (few tens of picosecond) due to efficient internal conversion as well as vibration relaxation, which enables individual dopant absorb more than ten photons within an excitation pulse. The above speculation has been confirmed by the consideration of heat-balance in time-of-flight mass spectroscopy ${ }^{37}$ transmittance measurement of excitation laser pulse, ${ }^{17}$ and computational and experimental studies on fluence dependence of fluorescence time profiles. ${ }^{16}$

This mechanism, however, is not applicable to the case of polystyrene ablation as discussed below. In the transmittance study (see Fig. 2), we showed that the absorbance of the polystyrene films did not change within the excitation pulse duration, while in the doped polymer ablation it changed considerably due to the effect of absorption by some transient species. ${ }^{15,17}$ Indeed, we can easily estimate the number of photons absorbed per a chromophore (phenyl ring) at a given fluence from the results of transmittance measurements. For the present threshold of $130 \mathrm{~mJ} / \mathrm{cm}^{2}$ it is estimated to be 0.1 , or in other words, only $10 \%$ of the phenyl rings are excited in the irradiated volume during the pulse. The result of numerical estimation should be 
critically distinguished from the case of doped polymer system in which each dopant can absorb more than ten photons at its threshold, ${ }^{16}$ namely, all the dopants would be repeatedly excited. In such case, it was demonstrated that temporal fluorescence profiles were changed with the laser fluence. We measured time-profiles of excimer fluorescence of the polystyrene films at various fluences, however, one can observe no fluence dependence of fluorescence time-profile.

As discussed above, the cyclic multiphotonic absorption mechanism should be eliminated for the polystyrene ablation. In the polystyrene, there exists a great deal of chromophores compared to doped polymer systems, therefore, the ground as well as excited singlet states of chromophores are hardly bleached even under the intense laser irradiation, and the number of the ground states is always higher than that of transient species.

The present results on $248 \mathrm{~nm}$ ablation of polystyrene are now compared with that of liquid benzene derivatives. As we have demonstrated already in the previous work, ablation of neat benzene derivatives such as toluene, benzyl chloride, etc. are brought about photochemically. ${ }^{13}$ These molecules are well known of their photochemical reactivity of $\beta$ bond cleavage which is possibly expected to take place in polystyrene. In the near liquid systems, molecular motion is quite easy, various molecular orientations are possible, the photochemical fragmentation have an appreciable yield, and the separation of the produced radicals is accelerated. On the contrary, in the solid polystyrene system such photochemical processes are relatively slow, and the reactive monomer states would undergo the quick deactivation to the excimer states, hence the photochemical ablation mechanism should be eliminated in the present case.

Finally, we briefly mention the preceding works on $248 \mathrm{~nm}$ ablation of polystyrene by other researchers. One characteristic among them is that the large discrepancy with the threshold value ranging from 35 to $250 \mathrm{~mJ} / \mathrm{cm}^{2}$. Lazare et al. determined the ablation threshold to be $35 \mathrm{~mJ} / \mathrm{cm}^{2}$ by means of micro-balance techniques. ${ }^{38}$ Almost the same value was obtained by Lemoine et al. using etchdepth measurements. ${ }^{26}$ On the other hand, Srinivasan et al. estimated it to be $\sim 200$ $\mathrm{mJ} / \mathrm{cm}^{2}$ by pyroelectric detection, ${ }^{39}$ and the largest one is $250 \mathrm{~mJ} / \mathrm{cm}^{2}$ determined photoacoustically by Paraskevopoulos et al. ${ }^{40}$ The present threshold of $130 \mathrm{~mJ} / \mathrm{cm}^{2}$ is between all the above values. It is noteworthy that our threshold is close to that of truncation of reflected excitation pulse $\left(120 \mathrm{~mJ} / \mathrm{cm}^{2}\right) .{ }^{40}$ The larger two values of threshold $\left(200\right.$ and $\left.250 \mathrm{~mJ} / \mathrm{cm}^{2}\right)$ may be too high, since we confirmed a slight morphological change at $180 \mathrm{~mJ} / \mathrm{cm}^{2}$ in the imaging experiment. For the lower value of $35 \mathrm{~mJ} / \mathrm{cm}^{2}$, temperature at the fluence was, by using eq. (1), calculated to be $110^{\circ} \mathrm{C}$ which is considerably lower than thermal degradation temperature $\left(\sim 300^{\circ} \mathrm{C}\right)$. If this is correct, the photothermal ablation would play a minor role in the ablation at the fluence, and a photochemical bond-cleavage may partly contribute to the ablation.

Anyway, although it seems difficult to give a precise interpretation to the scattering of threshold value, we have succeeded in clarifying the temperature at the threshold, photodynamics of emitting species as well as pyrolysis at the present stage. Quite recently the photothermal ablation of polystyrene was proposed also at $308 \mathrm{~nm}$ excitation. ${ }^{41}$ Also for this case, we believe that the primary photodynamics 
such as excimer formation and heat generating processes are almost similar to that discussed in this paper.

\section{SUMMARY}

In this work, we investigated the fundamental mechanism responsible for the 248 $\mathrm{nm}$ ablation of polystyrene film. Etch depth measurement and nanosecond imaging method provided us the ablation threshold with high accuracy. We estimated the temperature at the threshold by using the effective absorption coefficients which were confirmed by the time-resolved transmittance measurement. The temperature was in good agreement with that of thermal decomposition, based on which we proposed photothermal ablation. The results of imaging as well as spectroscopy were well interpreted in terms of the photothermal mechanism. The scattering of threshold suggests that nonlinear photochemical as well as photophysical processes of polystyrene around these fluences are quite complicated.

\section{Acknowledgement}

This work was partly supported by the Grant-in-Aid from the Ministry of Education, Science, and Culture of Japan (06239101 Priority-Area-Research "Photoreaction Dynamics" and 07554063). Thanks also to Prof. Hiroshi Miyasaka of Kyoto Institute of Technology for his helpful discussion on the photodynamics of polystyrene.

\section{References}

1. R. Srinivasan and W. J. Leigh. J. Am. Chem. Soc., 104, 6784 (1982).

2. Y. Kawamura, K. Toyoda and S. Namba. Appl. Phys. Lett., 40, 374 (1982).

3. R. Srinivasan, B. Braren, R. W. Dreyfus, L. Hadel and D. E. Seeger. J. Opt. Soc. Am., B3, 785 (1986).

4. M. Bolle, K. Luther, J. Troe, J. Ihlemann and H. Gerhardt. Appl. Surf. Sci., 46, 279 (1990).

5. H. Hiraoka, T. J. Chuang and H. Masuhara. J. Vac. Sci. Technol., B6, 463 (1988).

6. T. J. Chuang, H. Hiraoka and A. Modl. Appl. Phys., A45, 277 (1988).

7. R. Srinivasan, B. Braren and K. G. Gasey. J. Appl. Phys., 68, 1842 (1990).

8. G. Koren and J. T. C. Yeh. J. Appl. Phys., 56, 2120 (1984).

9. J. H. Brannon, J. R. Lankard, A. I. Baise, F. Burns and J. Kaufman. J. Appl. Phys., 58, 2036 (1985).

10. R. Srinivasan, B. Braren and R. W. Dreyfus. J. Appl. Phys., 61, 372 (1987).

11. R. Srinivasan and W. J. Leigh. J. Am. Chem. Soc., 104, 6784 (1982).

12. J. B. Birks. Photophysics of Aromatic Molecules, Wiley, London (1970).

13. Y. Tsuboi, H. Hatanaka, H. Fukumura and H. Masuhara. J. Phys. Chem., 98, 11237 (1994).

14. H. Masuhara, H. Hiraoka and E. E. Marinero. Macromolecules, 20, 450 (1987).

15. H. Fujiwara, H. Fukumura and H. Masuhara. J. Phys. Chem., 99, 11844 (1995).

16. H. Fukumura and H. Masuhara. Chem. Phys. Lett., 221, 373 (1994).

17. H. Fujiwara, H. Fukumura and H. Masuhara. Appl. Phys. Lett., 64, 2451 (1994).

18. Y. Tsuboi, H. Fukumura and H. Masuhara. Appl. Phys. Lett., 64, 2745 (1994).

19. H. Masuhara, S. Eura, H. Fukumura and A. Itaya. Chem. Phys. Lett., 156, 446 (1989); H. Masuhara, A. Itaya and H. Fukumura. ACS Symp. Ser., 412, 400 "Molecular Studies on Laser Ablation Processes of Polymeric Materials by Time-Resolved Luminescence Spectroscopy" (1989); H. Fukumura and H. Masuhara. J. Photopolym. Sci. Technol., 5, 223 (1992).

20. G. Koren. Appl. Phys. Lett., 50, 1030 (1987).

21. G. H. Pettit and R. Sauerbrey. Appl. Phys. Lett., 58, 793 (1991).

22. R. Srinivasan, B. Braren, K. G. Casey and M. yeh. Appl. Phys. Lett., 55, 2790 (1989). 
23. H. Fukumura, E. Takahashi and H. Masuhara. J. Phys. Chem., 99, 750 (1995).

24. D. E. Hare and D. D. Dlott. Appl. Phys. Lett., 64, 715 (1994).

25. R. Larciprete and M. Stuke. Appl. Phys., B42, 181 (1987).

26. P. Lemoine, W. Blau, A. Drury and C. Keely. Polymer, 34, 5020 (1993).

27. X. Wen, D. E. Hare and D. D. Dlott. Appl. Phys. Lett., 64, 184 (1994).

28. H. Fukumura, N. Mibuka, S. Eura and H. Masuhara. Appl. Phys., A53, 255 (1991).

29. J. Brandrup and E. H. Immergut. Polymer Handbook Third Edition. V/82, John Wiley \& Sons, Inc. (1989).

30. S. L. Madorsky and S. Strauss. J. Research of National Bureau of Standards, 40, 417 (1948).

31. J. Brandrup and E. H. Immergut. Polymer Handbook Third Edition. II/372, John Wiley \& Sons, Inc. (1989).

32. I. B. Berlman Handbook of Fluorescence Spectra of Aromatic Molecules, Academic Press, New York (1971).

33. H. Miyasaka, F. Ikejiri and N. Mataga. Chem. Phys. Lett., 202, 419 (1993).

34. H. Masuhara, S. Ohwada, Y. Seki, N. Mataga, K. Sato and S. Tazuke. Photochem. Photobiol., 32, 9 (1980).

35. H. Miyasaka, F. Ikejiri and N. Mataga. J. Phys. Chem., 92, 249 (1988).

36. R. W. B. Pearse and A. G. Gaydon. The Identification of Molecular Spectra, Wiley, London (1976).

37. H. Fukumura, N. Mibuka, S. Eura, H. Masuhara and N. Nishi. J. Phys. Chem., 97, 13761 (1993).

38. S. Lazare and V. Granier. J. Appl. Phys., 63, 2110 (1988).

39. P. E. Dyer and R. Srinivasan. J. Appl. Phys., 66, 2608 (1989).

40. G. Paraskevopoulos, D. L. Singleton, R. S. Irwin and R. S. Taylor. J. Appl. Phys., 70, 1938 (1991).

41. M. Tsunekawa, S. Nishio and H. Sato. Jpn. J. Appl. Phys., 34, 218 (1995). 